\title{
Michael Krekel \\ Vor 50 Jahren: Abschied vom ersten Bundeskanzler »Die dritte Entlassung war die schlimmste«
}

\author{
Notiz zur Zeitgeschichte
}

Kerzengerade und mit ernster Miene steht der Mann an seinem Platz, um die Würdigungen des Bundestagspräsidenten entgegenzunehmen - die Arme nach unten hängend, den Kopf zum Sprecher gewandt. Eugen Gerstenmaier ehrt in einer gut dreißigminütigen Ansprache Konrad Adenauer, der als Bundeskanzler von Beginn an der Bundesrepublik seinen Stempel aufdrückte - und mithin der Ära, die er repräsentierte, ihren Namen gab.

Am 15. Oktober 1963 würdigt der Deutsche Bundestag das Wirken des scheidenden Kanzlers im Rahmen einer Sondersitzung. Dessen Präsident lässt Verdienste und Leistungen des 87-jährigen noch einmal Revue passieren. Am Schluss erheben sich die Abgeordneten des Hohen Hauses von ihren Stühlen, bevor der Laudator zum Fazit ansetzt: »Konrad Adenauer hat sich um das Vaterland verdient gemacht.«

Tatsächlich steht die sachlich-nüchterne, aus dem antiken Rom stammende Formel kaum in einem angemessenen Verhältnis zur Summe dessen, was in Adenauers vierzehnjähriger Amtszeit geschaffen wurde. Die Bilanz ist beeindruckend: Die Bewältigung der Kriegsfolgen und der Wiederaufbau der Städte, die Erneuerung des Sozialstaates und die Integration von Millionen Vertriebener und Flüchtlinge, die Herausführung des westdeutschen Kernstaates aus dem Zustand der Besatzung und Fremdbestimmung auf das Niveau eines souveränen Staates, aus der außenpolitischen Isolation in die Gemeinschaft freiheitlich-demokratischer Völker, nicht zuletzt die Versöhnung mit den Nachbarn im Westen, allen voran Frankreich. Die wohl wichtigste Leistung - und zu wesentlichen Anteilen Adenauers Verdienst: Am Ende seiner Kanzlerschaft, weniger als zwei Jahrzehnte nach der Befreiung von der Hitler-Diktatur, war mit der Bundesrepublik Deutschland ein lebendiger demokratischer Staat mit funktionierenden Institutionen geschaffen worden, der von der westdeutschen Mehrheitsgesellschaft voll akzeptiert war.

\section{Gebengt, tief gebengt, aber nicht gebrochen}

Noch 1945, das Deutsche Reich lag in Trümmern, hatte der von der amerikanischen Besatzungsmacht wieder eingesetzte Kölner Oberbürgermeister Adenauer seine Antrittsrede vor der Kölner Stadtverordnetenversammlung mit den Worten beendet: »Gebeugt, tief gebeugt, aber nicht gebrochen «. Damals war es das vorrangige, alle Tagespolitik überragende Ziel, die unter den Kriegsfolgen leidenden Deutschen nicht nur phy- 
sisch-materiell, sondern auch seelisch und moralisch wieder aufzurichten: Eine Herkulesaufgabe, die durch die Lasten der Besatzung noch verschärft wurde.

Jetzt, im Herbst 1963, darf die westdeutsche Republik stolz sein auf das Erreichte und wieder aufrecht stehen. Vor allem die wirtschaftliche Aufbauleistung ist beeindruckend: Die Wachstumsraten sind hoch, die Arbeitslosenzahlen niedrig, der Export boomt wie kaum zuvor. Keine Frage: Im Jahre fünfzehn ihrer Existenz steht die Bundesrepublik, die weithin mit ganz Deutschland identifiziert wird, in der vordersten Reihe der bedeutendsten Wirtschaftsnationen. Längst gilt sie als das zugleich bestaunte und beneidete Land des Wirtschaftswunders, das bald den millionsten »Gastarbeiter « begrüßen wird. Damit, auch damit, hat man einen Großteil der Selbstachtung, auch den Respekt und die Anerkennung des Auslands zurück gewonnen.

Man mag den ökonomischen Erfolg in der Frühphase der Bundesrepublik großenteils Ludwig Erhard, dem »Vater des Wirtschaftswunders", zuschreiben, aber dies schmälert Adenauers Leistung keineswegs. Denn er war es, der, noch als Vorsitzender der Britischen Besatzungszone, in den Monaten nach der Währungsreform Person und Programm des liberalen Wirtschaftsprofessors in der CDU zur Geltung verhalf, dessen Reformkonzept der Sozialen Marktwirtschaft unterstützte und ihn, Erhard, nach Wahlsieg und Koalitionsbildung mit der eher marktwirtschaftlich orientierten FDP zum Bundeswirtschaftsminister machte.

Die Botschaft der Stunde im Bundestag lautet: Die noch vor wenigen Jahren daniederliegenden Deutschen können wieder aufrecht stehen und voller Zuversicht in die Zukunft blicken. Auch Adenauer benutzt das symbolträchtige Bild vor dem Plenum des Hohen Hauses: »Wir Deutschen dürfen unser Haupt wieder aufrecht tragen, denn wir sind eingetreten in den Bund der freien Nationen. « Er erinnert an die Ausgangslage des Jahres 1949, die »Trümmerfelder « und daran, »wie der deutsche Name damals in der Welt gewertet wurde und wie es heute anders geworden ist.« Dass der »deutsche Name im Ausland wieder seinen Klang hat «, sei nur möglich geworden durch das deutsche Volk, denn ohne dessen erfolgreiches Bemühen müsse »jedem Parlament und jeder Regierung « der Erfolg versagt bleiben. Adenauer trägt, wie fast immer, seine Worte frei vor. Er spricht über »die Last, die der moderne Fortschritt auf den Menschen legt « und die Wiedervereinigung - sie sei nur möglich » mit Hilfe unserer Freunde« und werde kommen, »wenn wir achtsam und vorsichtig und geduldig sind «. Wie Helmut Kohl, der sich ein gutes Vierteljahrhundert später im Park des Kanzleramts gegenüber Michael Gorbatschow in ähnlicher Weise äußern wird, ist er überzeugt: "Man kann einem Volke wie dem deutschen Volke nicht widersprechen und man kann ihm keinen Widerstand leisten, wenn es in Frieden seine Einheit wiederherstellen will.«

Zugleich mahnt der scheidende Regierungschef, das bisher Erreichte nicht zu gefährden und die wesentlichen Ziele - Sicherheit, Stabilität, Wohlfahrt - aus den Augen zu verlieren oder aufs Spiel zu setzen. Dabei erweisen sich genuin bürgerliche Tugenden, allen voran Berechenbarkeit, Verlässlichkeit und Stetigkeit als Schlüssel für erfolgreiches politisches Handeln: »Gerade für ein geschlagenes Volk, wie wir es waren, nach einem Krieg, der von Deutschen vom Zaun gebrochen war, ist die Stetigkeit in der Politik eine Grundbedingung und die Voraussetzung für das Ansehen eines Volkes.« 
Das Institut für Demoskopie Allensbach hat 1963 ermittelt, dass 62\% der Deutschen auf die Frage, wann es Deutschland im 20. Jahrhundert am besten gegangen sei, »in der Gegenwart, heute « zur Antwort geben. Die hohe Bewunderung, die der scheidende Kanzler, damals wie heute, in weiten Teilen der Öffentlichkeit genießt, hängt wesentlich damit zusammen, dass er den Landsleuten im Westen nicht nur zu Wohlstand, sondern $\mathrm{zu}$ internationalem Ansehen verholfen hat. Das bleibt nicht folgenlos in einer Zeit, die vor allem außenpolitisch durch Krisen geprägt ist und in der Sicherheit und Stabilität politische, gesellschaftliche und persönliche - einen besonders hohen Stellenwert besitzen. Bei regelmäßig durchgeführten Meinungsumfragen schneidet der Bundeskanzler sogar deutlich besser ab als sein berühmter Vorgänger, Reichskanzler Otto von Bismarck. Auf die Frage » welcher große Deutsche hat Ihrer Ansicht nach am meisten für Deutschland geleistet «, gaben 1963 60\% der Befragten den Namen Adenauers, 17\% den von Bismarcks an.

\section{Kanzler auf Zeit}

Der preußische Gründungskanzler, nicht die kurzlebigen Kanzler des Kaiserreichs und der Weimarer Republik, die Adenauer allesamt überlebt hat, galt bislang als Vergleichsmaßstab für staatsmännische Größe. Auch Gerstenmaier legt die Messlatte hoch. Er schlägt einen weiten Bogen hin zu Bismarck, in dessen Amtszeit Adenauer 1876 geboren worden war. Der Verweis auf dessen glanzlosen Rücktritt misslingt freilich: »Im Unterschied zu Ihnen«, so Gerstenmaier, zu Adenauer gewandt, »schied er im Unfrieden aus seinem Amt, so glanzvoll sein Abschied auch aussah«. Als dann der so Gerühmte vernimmt, »der einzige « zu sein, »der nach langer Regierungszeit unbesiegt und im Frieden von einem vergleichbaren Stuhle steigt«, gerät der Vergleich allerdings zur Peinlichkeit.

Denn »unbesiegt « und »im Frieden « war der zuweilen polarisierende, zuletzt in den eigenen Reihen umstrittene Kanzler nicht ausgeschieden. Mindestens seit 1959 war sein Stern im Sinken, und die potentiellen Nachfolger aus der Union warteten - wie lange eigentlich schon? - auf seine Ablösung. Das Ja und Nein in der Bundespräsidentenfrage (Adenauer erklärt sich zu einer Kandidatur bereit, zieht sich dann aber zurück) beschädigt sein Ansehen, auch sein nassforsches Vorpreschen im Streit um die Gründung des sogenannten Regierungsfernsehens kratzt an seinem Bild. Schließlich sein zögerliches Verhalten unmittelbar nach dem Bau der Berliner Mauer, das den Kanzler öffentliche Zustimmung und seiner Partei sechs Wochen danach Wählerstimmen kosten wird.

Bei der Bundestagswahl im September 1961 verliert die Union die absolute Mehrheit. Der 85-jährige Adenauer gerät nun vollends in die Defensive. Nach der Regierungsbildung formuliert er, vom liberalen Koalitionspartner bedrängt, jenen Brief an den damaligen CDU-Fraktions-vorsitzenden Heinrich Krone (mit Durchschlag an den FDPVorsitzenden Erich Mende), in dem es heißt, dass er, Adenauer, den Bundestagswahlkampf 1965 nicht mehr zu führen beabsichtige. Seither gilt er als »Kanzler auf Abruf«, dessen Amtszeit gnadenlos abläuft. 


\section{Der Kampf um die Nachfolge}

Gut ein Jahr später reißt die Spiegel-Krise auch den Regierungschef mit in den Strudel. Sie endet mit dem Ausstieg der fünf FDP-Minister und einer erneuten Regierungsbildung - der fünften und letzten unter einer Kanzlerschaft Adenauers. Fortan machen die innerparteilichen Gegner Ernst und blasen zum Angriff: Sie fordern ihren Vorsitzenden auf, die Kanzlerschaft endlich abzugeben und sich auf einen Rückzugstermin festzulegen.

Das geschieht Anfang Dezember 1962. Im Rahmen der Koalitionsgespräche erklärt der Kanzler, er beabsichtige, in den Wochen nach den Parlamentsferien des folgenden Jahres zurückzutreten. Damit wird die Regelung der Nachfolgefrage akuter denn je. Der Streit darüber wird Monate dauern - und mit der vollständigen Niederlage Adenauers enden.

Die wichtigsten Entscheidungen fallen in der Bundestagsfraktion. Dessen Vorsitzender Heinrich von Brentano wird Anfang März beauftragt, Gespräche über die Kandidatenauswahl zu führen. Dabei stellt sich schnell heraus, dass die Fraktion mit großer Mehrheit die »Wahllokomotive « Ludwig Erhard favorisiert. Die meisten, auch Adenauers engste Mitstreiter, setzen auf den populären Wirtschaftsminister. In seinem Urlaubsort Cadenabbia am Comer See muss Adenauer erfahren, dass die Zustimmungsrate zum Kandidaten in der Unionsfraktion bei etwa 70\% liegt. Die Würfel fallen Ende April. In der entscheidenden Fraktionssitzung erhält Erhard 159 der 225 abgegebenen Stimmen bei 47 Gegenstimmen und 19 Enthaltungen.

Das Ergebnis schmerzt »den Alten«, der sich mit Händen und Füßen gegen eine Nominierung Erhards gewehrt hatte. Erst die schleichende Verdrängung aus dem Amt, dann die Brüskierung in der Nachfolgefrage - das ganze kommt einer völligen Entmachtung gleich. In Wirklichkeit handelt es sich um einen parteiinternen Kanzlersturz, den ersten in der Geschichte der Bundesrepublik. Der kantige Kämpfer Adenauer, der als Oberbürgermeister im März 1933 von den Nationalsozialisten, im Oktober 1945 von britischen Militärs entlassen worden war, muss sich seinen Parteifreunden in der CDU beugen. Er kreidet es seiner "geliebten Partei«, so in einem Brief an Sohn Paul, an, ihn aus dem Amt gedrängt, ja gestürzt zu haben. Anlässlich seines Abschiedsempfang in der Beethovenhalle bekennt er gegenüber dem Publizisten Walter Henkels: »Ich gehe nicht frohen Herzens.«Später wird er vom Bundestagspräsidenten ähnlich vernommen. In Anspielung an seine beiden vorherigen Amtsentlassungen hört ihn Gerstenmaier sagen: »Die dritte Entlassung war die schlimmste.«

\section{Abseits der Macht}

Vor den Abgeordneten des Bundestages weiß der scheidende Regierungschef das Unvermeidliche mit Würde zu tragen. Seine Abschiedsworte sind knapp und ernst, enthalten aber auch jenes Quantum an Ironie und leisem Spott, ohne das Adenauer nicht Adenauer wäre. Er dankt »allen Mitgliedern« des Hohen Hauses (»natürlich den einen mehr, den anderen weniger«), selbst der Opposition, die »milder geworden ist« und ihre Pflicht erfüllt hat. Und er erinnert an jene Tage im Parlamentarischen Rat, als gemeinsam mit 
der SPD das konstruktive Misstrauensvotum vereinbart wurde. Für die eigene Partei oder die Fraktion indes hat er kein Wort übrig. Dann schreitet er unter langanhaltendem Beifall zu seinem Abgeordnetenplatz.

Jeder, der den Vollblutpolitiker Adenauer kennen gelernt hat, ahnt, wie schwer es ihm fallen wird, in die zweite Reihe zurückzutreten. Es beginnt die schlimme Zeit danach jenseits der Macht. Im Bundesratsflügel des Bundeshauses, Sitzungszimmer 119, bezieht er sein Büro, gibt Interviews und bäumt sich, meist erfolglos, dagegen auf, an den Rand geschoben zu sein. Noch ist er Abgeordneter, noch Vorsitzender seiner Partei, aber im Grunde verfügt er kaum über jenen Einfluss, der ihn auszeichnete, als er das Szepter noch in der Hand hielt. » Keiner hört mehr auf mich«, hört ihn seine Vertraute Anneliese Poppinga sagen, und der Adenauer-Biograph Hans-Peter Schwarz wagt den Vergleich mit einem Riesenwal, der aufs Trockene geschleudert wurde. Adenauer selbst fühlt sich so, als habe man ihm »Arme und Beine abgeschlagen«. Der Macht- und Vollblutpolitiker sieht sich ins politische Abseits gedrängt.

Dabei muss er zusehen, wie die Grundlagen seiner Außen- und Deutschlandpolitik einer harten Bewährungsprobe ausgesetzt sind. Da ist zum einen der Deutsch-Französische Vertrag, der durch die erzwungene Hinzufügung einer »transatlantischen « Präambel entwertet - mithin seines ursprünglichen politischen $Z$ wecks beraubt wird. Erhard, der auch persönlich Staatspräsident de Gaulle wenig abgewinnen kann, scheint jedenfalls nicht gewillt, in der Außenpolitik die französische Option mit Leben zu füllen. Schon deutet sich eine gefährliche innenpolitische Entwicklung an. Die Kluft zwischen jenen, die als »Atlantiker« eine engere Allianz unter Führung der Vereinigten Staaten fordern, und den Befürwortern des deutsch-französischen Zweibundes, den »Gaullisten «, beginnt sich zu öffnen. Andererseits bereitet dem Ex-Kanzler die sich vor allem im Gefolge der Berlin- und Kuba-Krisen anbahnende Zusammenarbeit zwischen den Supermächten Kummer. Das Ganze, so befürchtet er, geht letztlich zu Lasten deutscher Interessen und läuft auf eine Anerkennung des Status quo in Deutschland hinaus, also der staatlichen Teilung des Landes. Wo immer man in diesen Tagen hinschaut: Überall mischen sich Sorgen um den Fortbestand seines Werkes, vor allem in der Außenpolitik, einem Feld, auf dem er seinem Nachfolger wenig zutraut.

\section{Generationenwechsel}

In der Feierstunde des Bundestages werden solche Bedenken freilich nicht ausgesprochen. Im Mittelpunkt steht der Rückblick auf eine erfolgreiche Start- und Aufbauphase der zweiten deutschen Republik, die Adenauer wie kein anderer repräsentiert. Vor allem er war es, der dem jungen Staat auch jenseits der eigenen Grenzen zu einem positiveren Deutschlandbild verholfen hat. In jenen Tagen um den 15. Oktober treffen denn auch zahlreiche Schreiben aus dem Ausland im Kanzleramt ein, rund 75 darunter von Staatsund Regierungschefs. Soviel internationale Anerkennung ist noch keinem deutschen Staatsmann zuteil geworden. Einer der Bewunderer des Kanzlers, Israels Ministerpräsident David Ben Gurion, veröffentlicht in der Jerusalem Post einen Artikel unter der 
Überschrift The Greatness of Adenawer. Das Nachrichten-Magazin Der Spiegel, das Adenauer schon eine Sondernummer mit dem Titel Konrad Adenawers vierzehn Jabre gewidmet hat, überreicht einen stattlichen Lederband mit 43 Originalschreiben in- und ausländischer Wegbegleiter. Darunter befindet sich der Satz des Europäers Jean Monnet: »Ich glaube, dass die Geschichte ihn zu den großen Männern unserer Zeit rechnen wird."

Mit Adenauers Rücktritt geht diese Zeit unwiderruflich zu Ende. Er ist der letzte aus einer Reihe von Gründerfiguren, deren politische Karriere im Kaiserreich begann, in der Weimarer Republik reifte und in der Entstehungs- und Aufbaujahren der zweiten Republik ihren erfolgreichen Abschluss fand. Nun deutet sich ein Generationenwechsel an. Einige seiner Mit- und Gegenspieler aus der Gründergeneration sind bereits gegangen, in wenige Wochen werden andere folgen: Theodor Heuss, der erste Bundespräsident, Erich Ollenhauer, der parlamentarischer Gegenspieler, Heinrich von Brentano, der Außenminister und Fraktionsvorsitzende. An ihre Stelle treten Politiker, die alle mindestens eine Generation jünger sind als Adenauer. Sie können - und werden - auf dem aufbauen, was der Gründungskanzler hinterlassen hat. 